Proc. of the 13th Symposium of Magnetic Measurements and Modeling SMMM'2018, Wieliczka, Poland, October 8-10, 2018

\title{
Inverse Model of the Magnetic Hysteresis Based on Exponential Function
}

\begin{abstract}
W. Mazgaj*, Z. Szular And M. Sierzega
Institute of Electromechanical Energy Conversion, Cracow University of Technology, Kraków, Poland

Very often in numerical calculations of magnetic field distribution in ferromagnetic materials it is necessary to take into account a certain model of the magnetic hysteresis presenting changes of the flux density as a function of the magnetic field strength. However, in many cases it is profitable to apply an inverse model of the magnetic hysteresis describing the relationship between the field strength and the flux density. This paper presents a relatively simple method of approximation of field strength changes during magnetization of electrical steel sheets. It is assumed that the field strength changes are a sum or a difference of a function which describes one curve of the limiting hysteresis loop and a certain "transient" component. A method of the determination of functions describing changes of the field strength for assumed changes of the flux density is discussed in the paper. Theoretical considerations have been confirmed by comparison between measured and calculated hysteresis loop for chosen dynamo and transformer steel sheets.
\end{abstract}

DOI: $10.12693 /$ APhysPolA.136.724

PACS/topics: 75.50.Bb, 75.60.Ej

\section{Introduction}

The physics of the magnetic hysteresis phenomenon is quite well known [1-4]. In the range of small values of the magnetic field strength, reversible displacements of domain walls occur. The magnetization process of soft magnetic materials is irreversible for medium values of the field strength because this process is associated with irreversible movements of domain walls. The character of flux density changes (or magnetization changes) in the range close to the magnetic saturation is again reversible. In this case flux density vectors of individual domains rotate towards the vector of the magnetic field strength. Very often calculations of both the magnetic field distribution and eddy current distribution, as well as estimation of power losses in ferromagnetic materials require consideration of the magnetic hysteresis phenomenon. A chosen hysteresis model should fulfil the following conditions:

- model parameters should be easily determined,

- numerical calculations can be also carried out for magnetic field quantities containing a constant component (minor hysteresis loops),

- hysteresis model should have a reverse version that allows to compute changes of the field strength depending on the flux density changes,

- numerical calculation algorithm should be relatively simple,

- chosen model can be introduced into equations of the magnetic field distribution.

\footnotetext{
*corresponding author; e-mail: pemazgaj@cyfronet.pl
}

However, it should be emphasized that the most important criterion regarding the choice of the hysteresis model is the accuracy of calculation results in comparison to magnetic measurements. Creation of a suitable mathematical model on the basis of the physics of this phenomenon is still a relatively difficult problem, despite the fact that scientific literature contains a lot of papers presenting different mathematical models of this phenomenon. It has meaning especially in numerical calculations of the magnetic field distribution in dynamo or transformer steel sheets. The degree of difficulty increases significantly when a certain model of the hysteresis phenomenon should be taken into account in calculations of the field strength and flux density changes during the rotational magnetization. Different models of the magnetic hysteresis are used not only in calculations concerning axial magnetization processes, but they are also basis of vectorial models, which are applied in considerations of rotational magnetization processes. Some models describe the hysteresis phenomenon, presenting the magnetization as a function of the field strength $M=f(H)$; the other ones show the flux density as a function of the field strength $B=f(H)$.

Very often questions about the choice of an appropriate hysteresis model come up during the creation of a numerical program for field calculations. First of all, these questions relate to problems as the accuracy and times of numerical calculations. In many cases, a determination of parameters of a chosen hysteresis model is a serious problem. Some of these parameters are determined experimentally, others are based on theoretical considerations. However, the accuracy of modeling of real hysteresis changes is the fundamental criterion in assessment of the chosen hysteresis model. 


\section{Properties of hysteresis models}

Some hysteresis models are directly based on physical principles, primarily on energy relationships. The most known models which belong to this group are the Stoner-Wohlfarth model and the Jiles-Atherton model. The Stoner-Wohlfarth model is the oldest model of the hysteresis phenomenon, which describes the magnetization process as rotations of the magnetic moments of individual domains [5-7]. In this model the magnetization vector is a sum of magnetizations of single domains aligned along the specified directions. This model takes into account only the rotation of the magnetization vector, and the new position of this vector is determined on the basis of the minimum energy condition. However, the domain displacements, that are the fundamental mechanism in magnetization processes of ferromagnetic materials, are not taken into consideration. The StonerWohlfarth model is a vector model in fact. Applications of this model for macroscopic samples demand specification of a certain number of directions in the tested ferromagnetic material. Therefore, computation times are relatively long and they depend directly proportionally on the number of the specified directions. The resultant magnetization is the vector sum of the magnetizations of all specific directions. However, it is not possible to create an inverse model because the new position of the magnetization vector in this model depends on two input values, i.e., on the length of the field strength vector and its position with respect to the direction of the external field strength.

In the Jiles-Atherton model it is assumed that the energy supplied to the ferromagnetic material is a sum of the change of the magnetostatic energy and hysteresis losses [6-11]. In order to determine parameters of this model the following experimental values are needed (among others): the coercive force, the residual flux density, and the saturation parameters. Although the majority of these parameters are calculated on the basis of the measurement data, it is necessary to modify them in order to obtain the best representation of the real waveforms. The determination of a certain anhysteretic curve causes some difficulties because it is a theoretical curve; the authors of this model draw attention to this fact [8]. The basic version of the Jiles-Atherton model has to be modified accordingly in order to take into consideration an occurrence of minor hysteresis loops. The inverse model is formulated similarly to the base model [12]. The Jiles-Atherton model is suitable for modeling of relatively narrow hysteresis loops. It is not useful in cases when the hysteresis loop is relatively wide and especially has a rectangular shape. The main disadvantage of this model is the need to determine model parameters for almost each amplitude of the field strength. This fact definitely limits applications of this model in numerical calculation, especially for cases where the field strength amplitude is not known a priori. Nevertheless, this model is quite often used and even modified [13-15].
To hysteresis models based on energy dependences the Globus model should be included [7, 16]. In this model it is assumed that the magnetization process is based only on the mechanism of displacements of the domain walls. Despite a relatively simple algorithm and short calculation times, this model is rather unhelpful due to the unsatisfactory representation of hysteresis changes occurring in ferromagnetic materials. However, it should be emphasized that this model is practically a single model that uses energy dependences related directly to the domain structure of a ferromagnetic material.

The most known hysteresis model is the phenomenological Preisach model $[3,6,7,17]$. In this model it is assumed that the considered material consists of particles, so-called hysterons, magnetized to positive or negative saturation which have a rectangular hysteresis determined by two specific values. The Preisach triangle is a characteristic feature of this model. This triangle consists of two parts where one of these parts represents positively magnetized particles, and the second concerns negatively magnetized particles. The areas of these parts depend on changes in the field strength. A certain distribution function describes how many hysterons are located at a given point of the Preisach triangle. This model is built directly on the basis of the measurement data. The selection of a suitable distribution function is very time-consuming one. This model allows creating any hysteresis loops and any minor loops by the suitable choice of the distribution function. The inverse model is made similarly to the base model. The simple calculation algorithm is a significant advantage of this model, however, long calculation times that depend on a degree of the Preisach triangle discretization are significant disadvantages. The Preisach model allows describing the hysteresis changes for different ferromagnetic materials with relatively high accuracy. The Preisach model is still modified and often used in different applications, and it is a basis of some vectorial models of rotational magnetization [18-21].

Relatively simple model of the magnetic hysteresis, that allows us to approximate changes of the flux density is widely described in $[22,23]$. Any point $P$ with coordinates $(H, B)$ can move along a certain trajectory to one of the limiting magnetization curves $B=f(H)$, depending on the field strength changes (Fig. 1). It results from the phenomenon of the magnetic hysteresis considered in the macroscopic scale. In this model the flux density changes $B_{r}(H)$ for increasing values of the field strength $H$ and $B_{d}(H)$ for decreasing values $H$ are described by the following expressions [24]:

$B_{r}(H)=B_{b}(H)+\left[B_{0}-B_{b}\left(H_{0}\right)\right] \exp \left(-k_{H r}\left(H-H_{0}\right)\right)$,

$B_{d}(H)=B_{u}(H)-\left[B_{u}\left(H_{0}\right)-B_{0}\right] \exp \left(k_{H d}\left(H-H_{0}\right)\right)$,

where $B_{b}(H), B_{u}(H)$ are bottom and upper curves of the limiting hysteresis loop, respectively, $H_{0}, B_{0}$ are initial values of the hysteresis changes, $k_{H r}, k_{H d}$ are coefficients selected on the basis of some empirically measured minor hysteresis loops. 


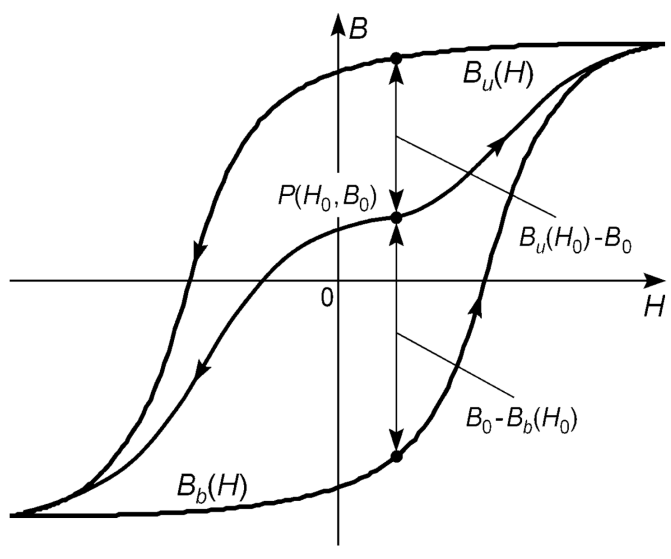

Fig. 1. Hysteresis loop as a function $B=f(H)$.

The second components, which contain exponential functions, can be treated as certain pseudo-transient components of changes of the flux density. The coefficients $k_{H r}, k_{H d}$ influence the "speed" of the point $P$ moving to one of curves of the limiting hysteresis loop. Values of the coefficients $k_{H r}, k_{H d}$ depend on the initial position of the point $P\left(H_{0}, B_{0}\right)$ with respect to the curves of the limiting hysteresis loop. The differences $B_{0}-B_{b}\left(H_{0}\right)$, $B_{u}\left(H_{0}\right)-B_{0}$ are initial values of "transient" components of the flux density changes.

On the one hand, this model allows to calculate flux density changes without going into details of the domain structure of the given ferromagnetic materials. On the other hand, this model directly uses the hysteresis properties which concern trajectories of any point $(H, B)$ inside the limiting hysteresis loop. The proposed approach to hysteresis modeling also allows to calculate changes of the flux density when a certain alternating field strength has a constant component [23]. The minor hysteresis loops are symmetric with respect to the center of the coordinate system $(H, B)$. It is in accordance with the so-called Madelung rules [25, 26]. Steady state occurs after several cycles of the field strength changes unlike to the "classical" Preisach model where this state takes place already after a first cycle of the field strength changes.

To less known and rarely used models of the magnetic hysteresis belong the Hodgdon model [6, 7], and the model proposed by Bogucki [27]. In the first one the dependence between the field strength and flux density is presented by means of a special differential equation. Applying this model, the relatively high accuracy can be obtained for the magnetic materials which have a narrow hysteresis loop. The determination of the Hodgdon model functions is time-consuming and requires many tests, especially some little changes of these model parameters influence considerably the shapes of the calculated loops. The Bogucki model is based on two non-linear first-order differential equations, presenting changes of both the magnetic field strength and flux density in ferromagnetic materials. It is assumed that the resultant field strength is a superposition of two field strength components where the first one depends on the anhysteretic curve and the second one is determined by the appropriate exponential function. However, the anhysteretic curve can be determined only theoretically, similarly as in the Jiles-Atherton model.

It is worth noting that some hysteresis models, the so-called dynamic models, take into account influence of eddy currents on flux density changes in the ferromagnetic materials. In these cases, shapes of the hysteresis loops, that depend strongly on the amplitude and frequency of the field strength, are wider than the loops of the static hysteresis. Measurements of these hystereses have been most often performed for sinusoidal currents. However, the dynamic hysteresis models are being used increasingly rarely because many electrical devices containing magnetic materials are supplied by semiconductor converters. This means that changes in both the field strength and flux density have higher order harmonics. The influence of the eddy currents on the magnetic field distribution is often taken into account by means of separate equations which are based on the differential or integral form of the Maxwell equations.

\section{Approximation of field strength changes}

The so-called inverse hysteresis models that present changes of the field strength as dependences of the flux density are often applied in calculations of the magnetic field distribution, especially using a finite element method. In such cases, an unknown variable in equations of the magnetic field distribution is the vector potential $\boldsymbol{A}$, which allows to determine changes of the flux density $\boldsymbol{B}$ based on the dependence $\boldsymbol{B}=\operatorname{rot} \boldsymbol{A}$. Therefore, the field strength components $H$ assigned to individual elementary segments of the magnetic field space, especially in relation to the ferromagnetic materials, have to be replaced by appropriate functions of the flux density $B$.

Considering an inverse model of the magnetic hysteresis, point $P$ with the coordinates $(B, H)$ can move to one of the limiting magnetization curves $H=f(B)$, depending on changes of the flux density (Fig. 2). When the flux density $B$ increases then changes of the field strength $H_{r}(B)$ can be written in the following form:

$H_{r}(B)=H_{u}(B)-\left[H_{u}\left(B_{0}\right)-H_{0}\right] \exp \left(-k_{B r}\left(B-B_{0}\right)\right), \quad(3)$ where $H_{u}(B)$ is upper curve of the limiting hysteresis loop (as a function $H=f(B)$ ), $H_{0}$ is initial value of the field strength, $B_{0}$ is initial value of the flux density, $k_{B r}$ is attenuation coefficient of the "transient" component when $B$ increases. For decreasing values of the flux density, the relationship describing changes of the field strength $H_{d}(B)$ can be written as follows:

$H_{d}(B)=H_{b}(B)+\left[H_{0}-H_{b}\left(B_{0}\right)\right] \exp \left(-k_{B d}\left(B-B_{0}\right)\right)$,

where $H_{b}(B)$ - lower curve of the limiting hysteresis loop (as a function $H=f(B)$ ), $k_{B d}$ - attenuation coefficient of the "transient" component, when $B$ decreases. 


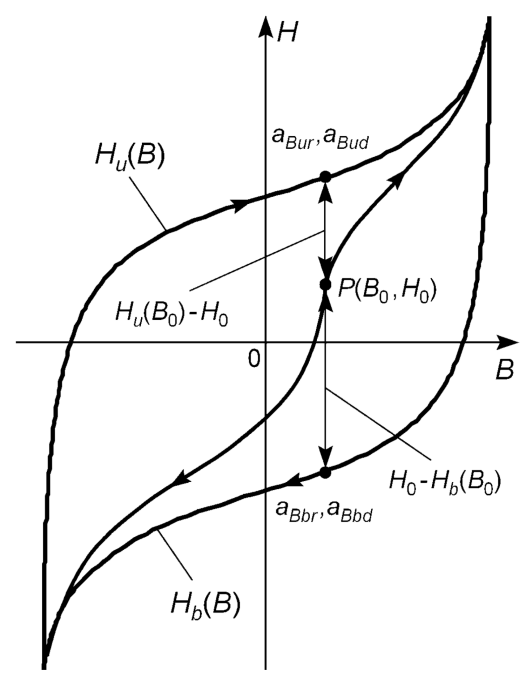

Fig. 2. Inverse hysteresis loops as a function $H=f(B)$.

The coefficients $k_{B r}, k_{B d}$ depend, first of all, on the initial position of the point $P\left(B_{0}, H_{0}\right)$ with respect to curves of the limiting hysteresis loop. In accordance to the physics of the hysteresis phenomenon, the derivative $\mathrm{d} H_{r} / \mathrm{d} B$ is in the range $\left(a_{B u r}, a_{B b r}\right)$ for the increasing values of the flux density $B ; a_{B u r}$ is equal to the value of this derivative when the initial point $P\left(B_{0}, H_{0}\right)$ is on the upper curve $H_{u}(B)$ of the limiting hysteresis loop, and $a_{B b r}$ is equal to this derivative when the point $P\left(B_{0}, H_{0}\right)$ is close enough to the bottom curve $H_{b}(B)$. The value $a_{B b r}$ is determined on the basis of several measured symmetric hysteresis loops. The coefficient $a_{B r}$ can be written in the following form:

$$
a_{B r}=a_{B b r}+\left(a_{B u r}-a_{B b r}\right) d_{B r}^{p},
$$

where $d_{B r}=\left[H_{0}-H_{b}\left(B_{0}\right)\right] /\left[H_{u}\left(B_{0}\right)-H_{b}\left(B_{0}\right)\right]$ (Fig. 2).

The coefficient $d_{B r}^{p}$ influences the "speed" of changes of the coefficient $a_{B r}$. The value of the exponent $p$ in relationship (5) can be greater or smaller than one and it should be experimentally determined for the tested ferromagnetic material, e.g., an electrical steel sheet. When we assume that $k_{H r}$ has a constant value in the small enough neighborhood of the point $B_{0}$, then for $B=B_{0}$ on the basis of (3) the following relationship can be written:

$$
\frac{\mathrm{d} H_{r}\left(B_{0}\right)}{\mathrm{d} B}=\frac{\mathrm{d} H_{u}\left(B_{0}\right)}{\mathrm{d} B}+k_{B r}\left[H_{u}\left(B_{0}\right)-H_{0}\right] .
$$

The derivative $\mathrm{d} H_{r}\left(B_{0}\right) / \mathrm{d} B$ has to be equal to $a_{B r}$, so the value $k_{B r}$ can be determined using the following relation:

$$
k_{B r}=\frac{a_{B r}-\frac{\mathrm{d} H_{u}\left(B_{0}\right)}{\mathrm{d} B}}{H_{u}\left(B_{0}\right)-H_{0}} .
$$

The value $a_{B r}$ for the initial point $P\left(H_{0}, B_{0}\right)$ is determined on the basis of (5) and next value of the coefficient $k_{B r}$ is calculated using (7). This value is inserted into relation (3) and for the next value of the flux density $B$, the new value of the field strength $H_{r}(B)$ is calculated.
When the value of the flux density $B$ decreases, then the coefficient $a_{B d}$ (with the similar meaning as $a_{B r}$ ) can be written in the following form:

$$
a_{B d}=a_{B u d}+\left(a_{B b d}-a_{B u d}\right) d_{B d}^{p},
$$

where $d_{B d}=\left[H_{u}\left(B_{0}\right)-H_{0}\right] /\left[H_{u}\left(B_{0}\right)-H_{b}\left(B_{0}\right)\right]$, $a_{B b d}$ is equal to the derivative $\mathrm{d} H_{d} / \mathrm{d} B$ when the point $P\left(H_{0}, B_{0}\right)$ is situated on the bottom limiting curve $H_{b}(B)$.

Similarly as previously, the value $a_{B u d}$ is determined on the basis of several measured symmetrical hysteresis loops. In this case, the derivative $\mathrm{d} H_{d} / \mathrm{d} B$ is in the range $\left(a_{B b d}, a_{B u d}\right)$ for the decreasing flux density $B$. Therefore, the coefficient $k_{B d}$ is determined similarly as $k_{B r}$ :

$$
k_{B d}=\frac{a_{B d}-\frac{\mathrm{d} H_{b}\left(B_{0}\right)}{\mathrm{d} B}}{H_{0}-H_{b}\left(B_{0}\right)} .
$$

The distances between the point $P$ and the curves of the limiting hysteresis loop are determined for initially assumed point $P\left(B_{0}, H_{0}\right)$. Depending on changes of the flux density $B$ the coefficients $a_{B b r}, a_{B u d}$ are calculated.
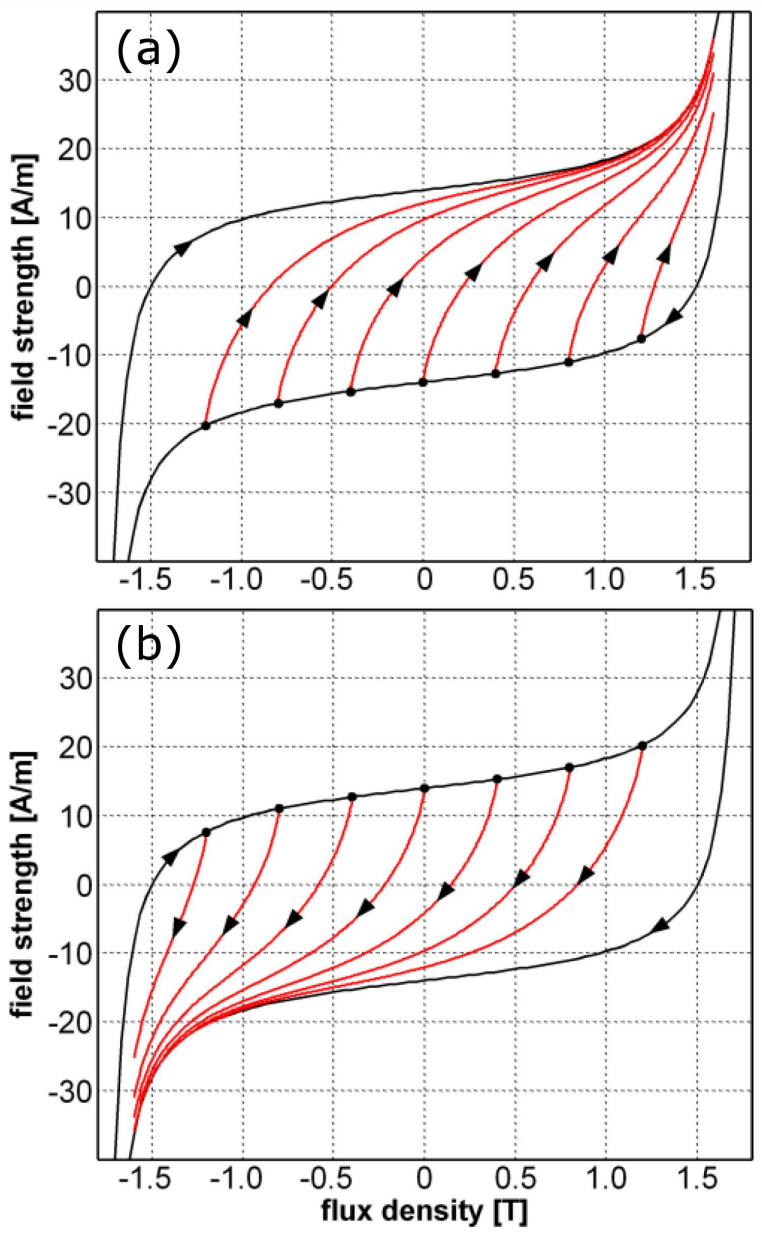

Fig. 3. Family of the first-reversal curves for: (a) increasing flux density value, (b) decreasing flux density value; ferromagnetic material - transformer sheet M120-27S. 




Fig. 4. Examples of minor loops determined using the inverse model of the magnetic hysteresis.
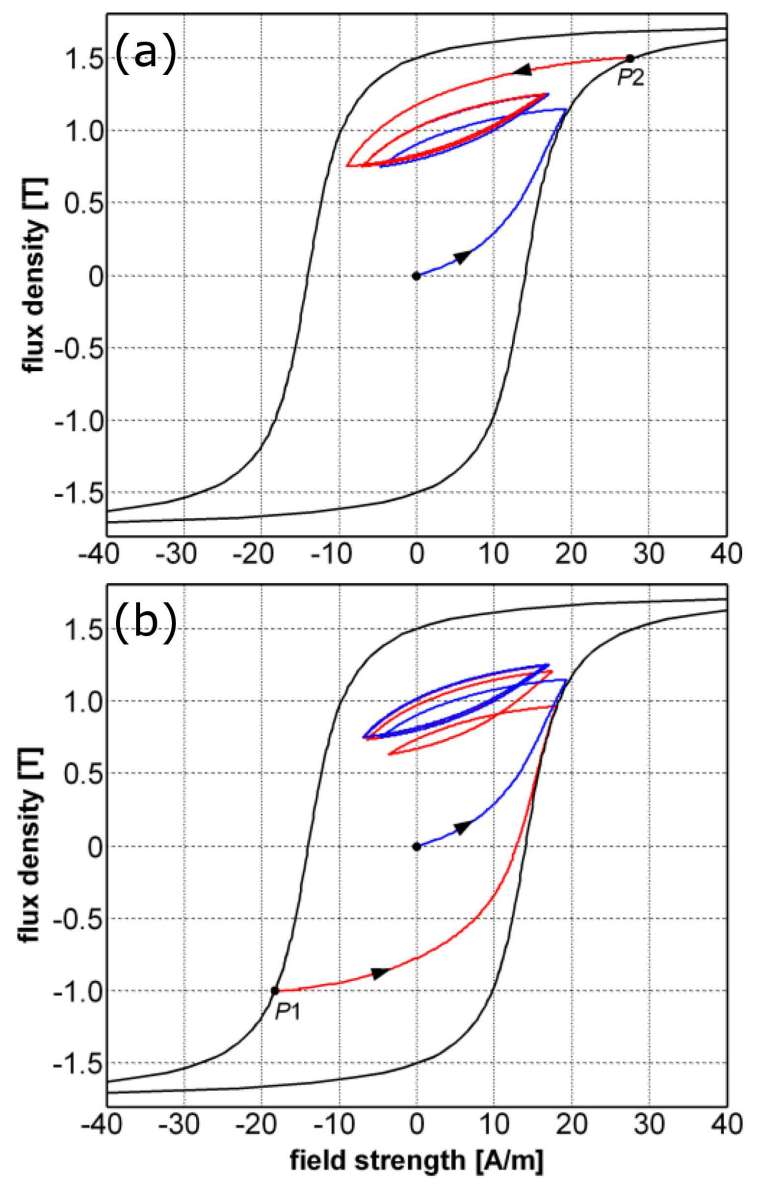

Fig. 5. Examples of minor loops, when the flux density has a constant component besides a variable component when the one starting point is in the center of the coordinate system and: (a) the second starting point $P 1$ is on the left curve of the limiting hysteresis loop, (b) the second starting point $P 2$ is on the right curve of the limiting hysteresis loop.
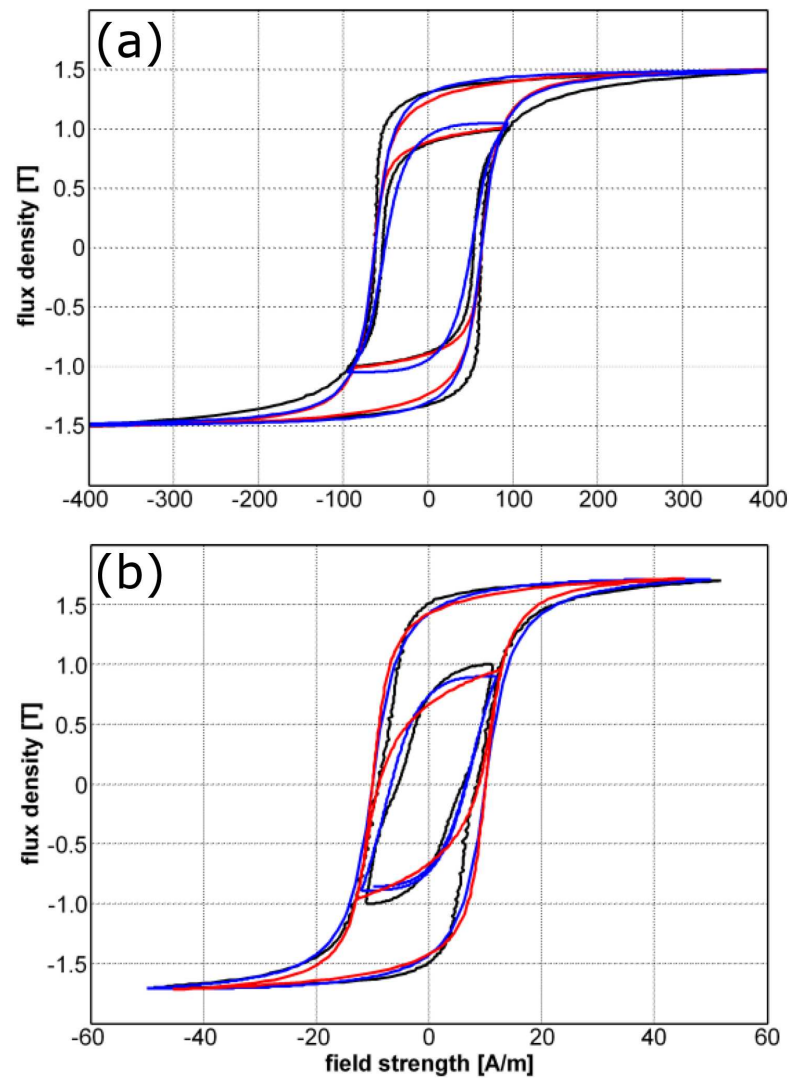

Fig. 6. Comparison of the measured and calculated hysteresis loops: (a) dynamo sheet M530-50A, (b) transformer sheet M120-27S; measured - black lines, calculated as $B=f(H)$ - blue lines, calculated as $H=f(B)$ — red lines.

Then the coefficients $a_{B r}, k_{B r}$ or $a_{B d}, k_{B d}$ are determined and a new value of the field strength $H_{r}$ or $H_{d}$ is calculated. Figure 3 shows a family of the first-reversal-curves for increasing and decreasing values of the flux density. In turn, Fig. 4 presents minor loops for different changes of the flux density. These loops are determined using the proposed inverse model.

Figure 5 presents examples of minor loops, when the flux density has a constant component. Regardless of the starting point, the minor loops in the steady states have to be the same. These states of the minor loops occur after several cycles of the flux density changes, unlike the classical Preisach model, where this state is achieved after a single cycle of the flux density (or field strength) changes.

In the proposed inverse model of the magnetic hysteresis any trajectory is uniquely determined by the coordinates of the starting point (Fig. 3). It fulfills the first one of Madelung's rules. The second one of these rules says that any hysteresis loop is closed (the so-called "returnpoint-memory"). Corresponding points on the minor loops are symmetrical with respect to the center of the coordinate system, i.e., points $A 1$ and $A 2, B 1$ and $C 2$, $B 2$ and $C 1$, and so on. However, it takes place only in 



Fig. 7. Comparison of the measured and calculated hysteresis loops: (a) ferrite $\mathrm{Mn}-\mathrm{Mg}$, (b) ferromagnetic material YuN14DK24; measured loops - black lines, calculated as $B=f(H)$ - blue lines, calculated as $H=f(B)$ - red lines.

the steady state of the minor loops (Fig. 4), because they drift gradually to this state. This is confirmed among others in $[28,29]$. The minor loops in the steady states determined for assumed changes of the flux density are the same independently of the coordinates of the starting point (Fig. 5). It refers to the third Madelung rule ("congruency property"). Obviously, the hysteresis loops for only alternating changes of the flux density are symmetric with respect to the center of the coordinate system (fourth rule).

Calculations with the use of the inverse model of the magnetic hysteresis were made for different electrical steel sheets. For example, Fig. 6a shows the comparison between measured and calculated hysteresis loops of the dynamo sheet M530-50A, and Fig. 6b presents a similar comparison regarding the loops of the transformer sheet M120-27S. In turn, Fig. 7 presents comparison of the measured and calculated hysteresis loops for ferrite $\mathrm{Mn}-\mathrm{Mg}$ [30] and for ferromagnetic material YuN14DK24, that is applied in hysteresis motors.

\section{Conclusions}

The field strength changes as the dependence of the flux density are written in terms of simple formulae. Therefore, they may be relatively easily inserted into equations of the magnetic field distribution. The times of numerical calculations are shorter than in other models. In order to apply this method the limiting hysteresis loop of the given electrical steel sheet has to be known. Additionally, some minor loops should be measured to choose the attenuation coefficients of the "transient" components correctly.

It is worth underlining that satisfactory calculation results can be obtained when the attenuation coefficients of the "transient" components have the same and constant values. As a result of this, calculation times can be significantly shortened. This is especially important in calculations of the magnetic field when the hysteresis phenomenon has to be taken into account in a large number of elementary segments resulting from the division of the magnetic field space.

The hitherto proposed models of the magnetic hysteresis refer to the "classical" shape of the hysteresis loop, but almost none of them show how to approximate changes of both the flux density and field strength in transformer sheets for magnetization directions other than the rolling direction. The proposed hysteresis model with some modifications can be used in studies of magnetization processes in these sheets [28].

The authors hope that this paper will encourage physicists to discuss on the formulation of the hysteresis model that will be based on physical, not just magnetic, properties of the ferromagnetic materials, especially that since two years the authors have tried to take into consideration the crystallographic structure of electrical steel sheets in the modeling of magnetization processes.

\section{Acknowledgments}

The research presented in this paper was funded by subsidies on science granted by Polish Ministry of Science and Higher Education under the theme No. E-2/731/2018/DS "Modelling of magnetization processes in magnetic circuits of electrical machines and devices for diagnostics and loss estimation".

\section{References}

[1] F. Brailsford, Physical Principles of Magnetism, Van Nostrand, London 1966.

[2] D.C. Jiles, Introduction to Magnetism and Magnetic Materials, Chapman \& Hall, London 1998.

[3] G. Bertotti, I.D. Mayergoyz, The Science of Hysteresis, Vol. I, Elsevier, Oxford 2006.

[4] S. Tumański, Handbook of Magnetic Measurements, CRC/Taylor \& Francis, Boca Raton 2011.

[5] L.D. Atherton, J.R. Beattie, IEEE Trans. Magn. 26, 3059 (1990). 
[6] A. Iványi, Hysteresis Models in Electromagnetic Computation, Akadémiai Kiadó, Budapest 1997.

[7] F. Liorzou, B. Phelps, D.L. Atherton, IEEE Trans. Magn. 36, 418 (2000).

[8] D.C. Jiles, D.L. Atherton, J. Appl. Phys. 55, 2115 (1984).

[9] K.H. Carpenter, IEEE Trans. Magn. 27, 4404 (1991).

[10] D.C. Jiles, IEEE Trans. Magn. 28, 2602 (1992).

[11] D.C. Jiles, J. Magn. Magn. Mater. 242, 116 (2002).

[12] N. Sadowski, N.J. Batistela, J.P.A. Bastos, M. LajoieMazenc, IEEE Trans. Magn. 38, 797 (2002).

[13] N.L. Mi, R. Oruganti, S.X. Chen, IEEE Trans. Magn. 34, 1294 (1998).

[14] H.L. Toms, R.G. Colclaser, M.P. Krefta, IEEE Trans. Magn. 37, 982 (2001).

[15] J. Izydorczyk, IEEE Trans. Magn. 42, 3132 (2006).

[16] M. Guyot, A. Globus, Phys. Status Solidi B 59, 447 (1973).

[17] I.D. Mayergoyz, Mathematical Models of Hysteresis, Springer-Verlag, New York 1991.

[18] V. Basso, IEEE Trans. Magn. 34, 2207 (1998).

[19] E.D. Torre, Magnetic Hysteresis, IEEE Press, New York 1999.
[20] E. Cardelli, G. Finocchio, E. Pinzaglia, IEEE Trans. Magn. 40, 892 (2004).

[21] A. Stancu, L. Stoleriu, P. Postolache, M. Cerchez, IEEE Trans. Magn. 40, 2113 (2004).

[22] W. Mazgaj, Arch. Electr. Eng. 216, 147 (2006).

[23] W. Mazgaj, Calculation of magnetic field distribution in soft magnetic materials taking into account hysteresis and anisotropy, monograph, vol. 379, Cracow University of Technology, Kraków 2010 (in Polish).

[24] W. Mazgaj, Z. Szular, M. Sierżega, Int. Symp. on Electrical Machines (SME), Andrychów (Poland), 2018, IEEE.

[25] S.E. Zirka, Y.I. Moroz, IEEE Trans. Magn. 35, 2090 (1999)

[26] K. Cao, R. Li, Sensors 19, 352 (2019).

[27] M. Bogucki, Archiwum Elektrotechniki XXX, 799 (1981).

[28] L.A.L. de Almeida, G.S. Deep, A.M.N. Lima, H. Neff, IEEE Trans. Magn. 39, 523 (2003).

[29] D.C. Jiles, Y. Melikhov, in: Handbook of Magnetism and Advanced Magnetic Materials, Wiley, 2007.

[30] C. Heck, Magnetic Materials and Their Applications, Butterworths, London 1974. 